\title{
DETERMINATION OF FLAVONOIDS IN STAMEN, GYNOECIUM, AND PETALS OF Magnolia grandiflora L. AND THEIR ASSOCIATED ANTIOXIDANT AND HEPATOPROTECTION ACTIVITIES
}

\author{
Nadia M. Sokkar ${ }^{\mathrm{a}, \mathrm{b}, *}$, Mohamed A. Rabeh${ }^{\mathrm{b}}$, Gamal Ghazal ${ }^{\mathrm{c}}$ and Amani M. Slem ${ }^{\mathrm{d}}$ \\ aNatural Products and Alternative Medicine Dept., Faculty of Pharmacy, King Abdulaziz University, Jeddah, 80200, Kingdom of \\ Saudi Arabia \\ 'Pharmacognosy Dept., Faculty of Pharmacy, Cairo University, Cairo, 11562, Egypt \\ 'National Organization for Drug Control and Research, 6 Abou-Hazem Street, Pyramids Ave. P.O.B. 29, Giza, Egypt \\ ${ }^{\mathrm{d} N a t i o n a l ~ R e s e a r c h ~ i n s t i t u t e ~ D o k k i, ~ G i z a, ~ E g y p t ~}$
}

Recebido em 04/10/2013; aceito em 29/01/2014; publicado na web em 27/03/2014

\begin{abstract}
Chromatographic analysis of flavonoids in ethyl acetate fractions of the stamen, gynoecium, and petal of Magnolia grandiflora L. by HPLC-PDA-MS/MS-ESI in the negative ionization mode was performed in this study. The results revealed the presence of eight flavonoids: apigenin 8-C-glucoside, luteolin 8-C-glucoside, quercetin 3-O-rutinoside, quercetin 3-O-galactoside, quercetin, 3-O-glucoside, kaempferol 3-O-rutinoside, isorhamnetin 3-O-glucoside, and isorhamnetin. Their quantification revealed that luteolin 8-C-glucoside is the major flavonoid and that the total phenolic content is concentrated primarily in the stamen. The antioxidant and hepatoprotective effects of ethanolic extract of the flower organs were evaluated against hepatotoxicity induced by $\mathrm{CCl}_{4}$, compared with the effects of silymarin.
\end{abstract}

Keywords: Magnolia grandiflora L.; antioxidant flavonoids; hepatoprotection.

\section{INTRODUCTION}

Magnolia is a genus comprising approximately 120 species and belongs to the family Magnoliaceae. Magnolia grandiflora L., commonly known as the Southern Magnolia or bull bay, is native to the Southeastern United States and is cultivated in many Mediterranean countries, including Egypt. Phytochemical investigations of the flower have resulted in the identification of flavonoids, ${ }^{1}$ sesquiterpenes, ${ }^{2}$ and volatile oils. ${ }^{3-8}$ Preparations from the flower have been extensively documented in traditional Chinese medicine. ${ }^{9}$ Biological studies on the extract of the flower have been conducted to investigate its effects on the cardiovascular system. ${ }^{2,10}$ The antimelanogenic, antioxidant, antimicrobial, ${ }^{3,11}$ and anticancer effects of the volatile constituents have also been investigated. ${ }^{5}$ Despite the importance of the flower of M. Grandiflora, little information about its flavonoids is available. Hence, in the present investigation, we used HPLC-PDA-MS/MSESI to investigate the flavonoid content in the extracts of the stamen, gynoecium, and petal of Magnolia grandiflora cultivated in Egypt and studied the extracts' antioxidant and hepatoprotective effects.

\section{EXPERIMENTAL}

\section{Standards and reagents}

Vitexin, orientin, rutin, nicotiflorine, hyperoside, and isorhamnetin were kindly supplied by the Pharmacognosy Dept., Faculty of Pharmacy, Cairo University. Folin-Ciocalteu reagent was obtained from Fluka (Switzerland). 2,2-Diphenyl-1-picrylhydrazyl (DPPH) was purchased from Sigma-Aldrich (Chemie GmbH, Steinheim, Germany). Silymarin was purchased from Grand Pharma Co., and chemicals for biochemical work were purchased from Sigma, BDH, and Merck (Darmstadt, Germany).

*e-mail: nadiasokkar@yahoo.com

\section{Solvents}

Methanol, formic acid, and acetonitrile HPLC-grade solvents were purchased from Merck. All other chemical reagents and solvents were analytical grade (DBH). Double-distilled water was used in the HPLC mobile phase.

\section{Plant material}

The white flowers of $M$. grandiflora L. were properly collected from Wally's private farm, Giza, Egypt in 2011. A sample was authenticated by Dr. Mohammad El-Gibali, senior botanist at the National Research Centre, Giza, Egypt. Voucher specimens of the separate floral parts (M-545) were deposited in the herbarium of Pharmacognosy Dept., Faculty of Pharmacy, Cairo University. The parts of the flower under investigation, i.e., the stamen, gynoecium, and petal, were air-dried and ground. The powders (120 g each) were defatted with petroleum ether for $24 \mathrm{~h}$, and the marc of each organ was separately macerated in $70 \%$ ethanol until exhaustion. The concentrated residues of the stamen (EES), gynoecium (EEG), and petal (EEP) were used for the biological experiments.

\section{General equipment}

The mass detector was performed on Thermo LCQ Advantage Max ion trap mass spectrometer (Thermo Finnigan, San Jose, CA, USA). The analysis was performed using the following settings: the gas (air) was heated to $400{ }^{\circ} \mathrm{C}$, capillary voltage was $4 \mathrm{kV}$, nebulizer gas was air, curtain gas was $\mathrm{N}_{2}$, collision gas was He, ionization was performed in negative mode $[\mathrm{M}-\mathrm{H}]^{-}$, and collision energy was 35 . The full-scan mass infusion was performed using a syringe pump (Hamilton syringe, $500 \mu \mathrm{L}$ ) directly connected to the electrospray ionization unit and operated at a flow rate of $10 \mu \mathrm{L} \mathrm{mL}^{-1}$. The technique used in LC-MS/MS was the total ion mapping experiment, where product ion scans for each parent ion were obtained to determine which parent ions lost a particular fragment to yield a particular product ion. 
HPLC analyses were performed using a PDA detector and an Intersil ODS- 2 C18 column $\left(2.1 \times 50 \mathrm{~mm}^{2}\right.$, particle size $3 \mu \mathrm{L}$, Alltech). The solvent system consisted of (A) $0.1 \%$ formic acid in water and (B) acetonitrile:methanol $(60: 40 \mathrm{v} / \mathrm{v})$. The gradient program was $70 \% \mathrm{~A}$ (0-2 min), 50\% A (2-6 min), 30\% A (6-9 min), 70\% A (9-12 min); flow rate was $0.2 \mathrm{~mL} \mathrm{~min}^{-1}$, and injection volume was $20 \mu \mathrm{L}$. The Xcalibur software (version 1.4) linked to the instrument was used to calculate the corresponding concentrations. TLC was performed on plates precoated with silica gel F254 (Fluka) (Merck, Germany). The TLC solvent systems for flavonoids were ethyl acetate:water:formic acid (85:15:10 v/v) and hexane:ethyl acetate:formic acid (20:19:1 $\mathrm{v} / \mathrm{v}$ ). The flavonoid spots were visualized under UV light (254 and $366 \mathrm{~nm}$ ) before and after they were exposed to ammonia vapor or sprayed with natural products (NP) reagent.

\section{Sample preparation for TLC and HPLC}

Air-dried powdered samples of the stamen, gynoecium, and petal of $M$. grandiflora ( $5 \mathrm{~g}$ each) were defatted with petroleum ether for $24 \mathrm{~h}$. The marc of each organ was extracted with $70 \%$ methanol by sonication at room temperature $(1 \mathrm{~h})$ and was subsequently filtered and concentrated under reduced pressure. The concentrated residues were separately suspended in water $(50 \mathrm{~mL})$ and fractionated between chloroform and ethyl acetate $(5 \times 100 \mathrm{~mL}$, each). The concentrated ethyl acetate fraction of each organ was accurately dissolved in 10 $\mathrm{mL}$ of methanol (HPLC grade) for the chromatographic analysis.

\section{HPLC-PDA-LC-MS/MS quantification of flavonoids}

The sample solutions were injected into the mass detector against reference standards of vitexin, orientin, rutin, nicotiflorine, hyperoside, and isorhamnetin (0.031-2 $\mathrm{mg} \mathrm{mL}^{-1}$, each). The stock solutions were stored at $-20{ }^{\circ} \mathrm{C}$. The stock and standard solutions were filtered through 0.45 - $\mu \mathrm{m}$ filters before being injected into HPLC and were diluted as necessary with methanol. Each concentration of the standards was analyzed in triplicate. Quantification of the flavonoid compounds was achieved by measuring the peak area against six concentrations of the standards and plotting standard curves; the concentrations of the compounds were determined as the mean values of three replicate injections. Quantitative determinations of vitexin, orientin, rutin, and nicotiflorine were performed as vitexin, orientin, rutin, nicotiflorine; hyperoside and isoquercetrin were determined as hyperoside; and isorhamnetin 3-O-glucoside and isorhamnetin were determined as isorhamnetin. Linear regression analysis for the curve was performed to determine $y=a x+b$, where $a$ and $b$ are the coefficients of the regression equation, $x$ is the concentration of the

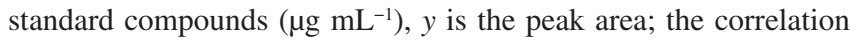
coefficient $r$ was also calculated.

The limit of detection was calculated as the concentration corresponding to three times the intensity of the background noise. The precision tests were performed by injecting each of the sample solutions six times. Measurements of the intra- and inter-day variability were used to determine the repeatability of the developed method. The intra-day variability was determined by analyzing each sample three times within the same day, and the inter-day reproducibility was performed on three different days. Relative standard deviation (RSD) was considered as the measure of precision.

\section{Colorimetric determination of total phenols}

A stock solution was prepared from the concentrated residues of another $70 \%$ methanol extract of the air-dried and defatted powdered stamen, gynoecium, and petal; the powders were dissolved in distilled water to a concentration of $1 \mathrm{mg} \mathrm{mL}^{-1}$. Polyphenols were determined using the Folin-Ciocalteau colorimetric method ${ }^{12}$ and expressed as $\mathrm{mg}$ of gallic acid equivalents $\mathrm{g}^{-1}$ of the dry plant material. The standard solution was prepared by dissolving $1 \mathrm{mg}$ of gallic acid in $100 \mathrm{~mL}$ of water. A calibration curve was constructed in the range of $2-10 \mu \mathrm{g} \mathrm{mL}^{-1}$ by diluting the stock solution in water. The stock and standard solutions ( $1 \mathrm{~mL}$ each) were separately mixed with 0.25 $\mathrm{mL}$ of Folin-Ciocalteau reagent and diluted to $25 \mathrm{~mL}$ using $290 \mathrm{~g}$ $\mathrm{L}^{-1}$ sodium carbonate in water. The absorbance was measured after $30 \mathrm{~min}$ at $730 \mathrm{~nm}$ against a blank prepared simultaneously using 0.5 $\mathrm{mL}$ of water as a compensating liquid.

\section{Determination of median lethal dose (LD50)}

The (LD50) of the hydro-ethanolic extracts of the stamen, gynoecium, and petal of $M$. grandiflora was estimated according to the method of Lorke. ${ }^{13}$

\section{In vitro antioxidant effect}

The antioxidant effect of the hydro-ethanolic extracts of the stamen, gynoecium, and petal was measured using the stable radical of 2,2-diphenyl-1-picryl-hydrazyl (DPPH). ${ }^{14}$ The prepared test samples (4 $\mathrm{mg} \mathrm{mL}^{-1}$ methanol, each) and silymarin $\left(2 \mathrm{mg} \mathrm{mL}^{-1}\right)$ were added in equal volumes (10 $\mu \mathrm{L}$ each) to a methanolic solution of DPPH (190 $\mu \mathrm{L})$ and incubated in the absence of light at room temperature for 30 min. The absorbance values were measured against methanol at 517 $\mathrm{nm}$ on a spectrophotometer (Unicam UV-VIS spectrophotometer, Japan). All tests were performed in triplicate. The inhibition (\%) was calculated according to the formula:

$\%$ of inhibition $=(1-$ Test absorbance $/$ Control absorbance $\times 100)$

\section{Hepatoprotective effect}

The hepatoprotective effect of the hydro-ethanolic extracts of the stamen, gynoecium, and petal (100 $\mathrm{mg} \mathrm{kg}^{-1}$ body weight, each) were measured according to the method of Klassen and Plaa. ${ }^{15}$ Intraperitoneal injection of $25 \% \mathrm{CCl}_{4}$ in liquid paraffin $\left(5 \mathrm{~mL} \mathrm{~kg}^{-1}\right)$ was used to induce liver toxicity in adult male albino Sprague-Dawley rats (120-155 g). The serum alanine aminotransferase (ALT), aspartate aminotransferase (AST), and alkaline phosphatase (ALP) were measured at time intervals of zero and two weeks before toxicity induction and $72 \mathrm{~h}$ and two weeks after $\mathrm{CCl}_{4}$ injection. The results were compared with that of a silymarin reference $\left(25 \mathrm{mg} \mathrm{kg}^{-1}\right.$ body weight). The negative control was considered for liver damaged rats receiving a daily dose of $1 \mathrm{~mL}$ saline.

\section{Statistical analysis}

The results were statistically analyzed using the Statistical Package for Social Sciences (SPSS, version 17; SPSS Inc., Chicago) for Windows, followed by Dennett's multiple comparison test (DMCT); $\mathrm{P}<0.05$ was considered as significant.

\section{RESULTS AND DISCUSSION}

Qualitative detection of the flavonoids in the ethyl acetate fraction was performed by TLC and subsequently by HPLC-PDA. The HPLC chromatogram (Figure 1) revealed that methanolic acetonitrile with $0.1 \%$ formic acid gave the best separation of the flavonoid peaks. ${ }^{16}$ In the present work, a gradient flow was used to elute the polar compounds with reasonable resolution. The mobile 

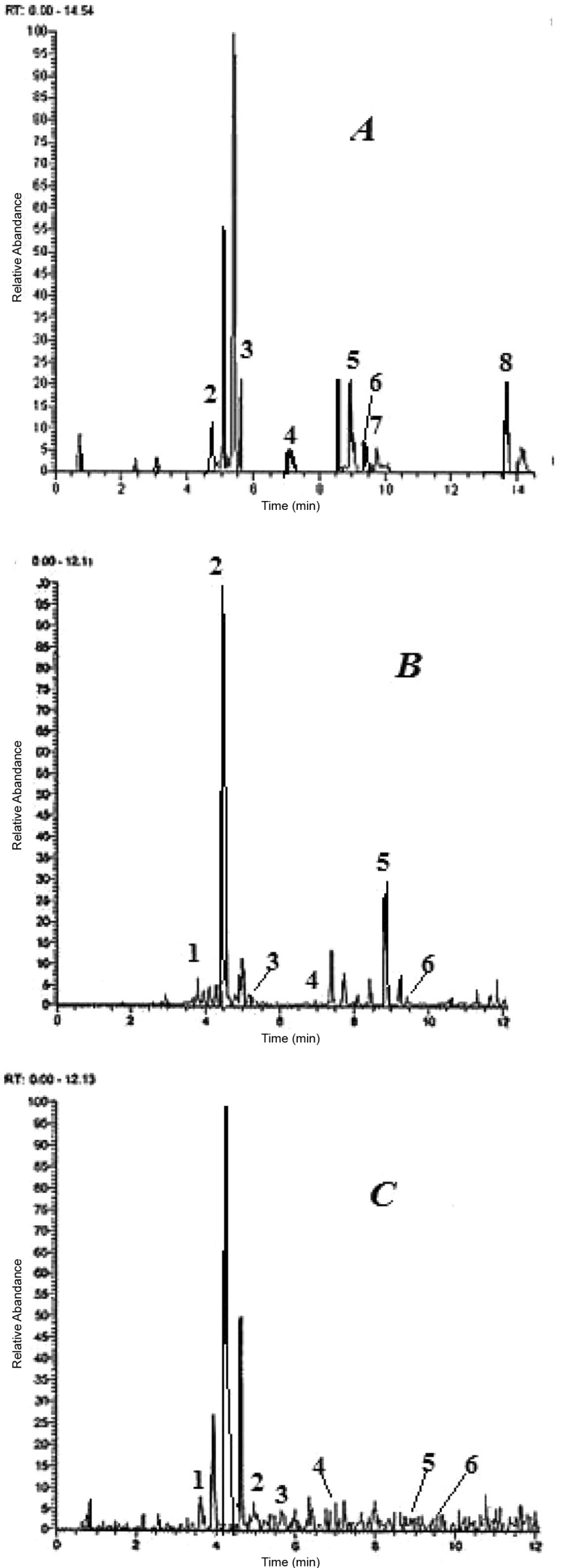

Figure 1. HPLC/PDA chromatogram of ethyl acetate extract of floral parts of M. grandiflora: A, stamen; B, gynoecium; $C$, petal phase applied in this experiment was the most suitable for the investigation of the $C$-glycosides vitexin (1) and orientin (2), where their peaks showed a relative retention greater than 1 . They were characterized on the bases of their product ion spectra, comparisons with reference samples, and literature data. ${ }^{17}$ TIC of LC-MS $(n=$ 2) chromatograms detected another six flavonols: $O$-glycosides (3-7) and $O$-methylated aglycone (8). A comparison of the retention times, UV and mass spectra of the deprotonated molecule $[\mathrm{M}-\mathrm{H}]^{-}$of glycosides and the ion corresponding to the deprotonated aglycone $[\mathrm{A}-\mathrm{H}]^{-}$with data compiled for known standards confirmed their identification as rutin (3), nicotiflorine (4), hyperoside (5), isoquercetrin (6), and isorhamnetin (8). Isorhamnetin (8) exhibited a specific fragmentation because of the loss of 15 units of the methyl radical to give $\mathrm{m} / \mathrm{z}, 300$. The identification of compound 7 was supported by the loss of 162 units from the pseudomolecular ion $m / z 477[\mathrm{M}-\mathrm{H}]^{-}$. The $\mathrm{MS}_{2}$ product-ion analysis of the parent ion provided a fragmentation pattern of isorhamnetin glucoside, similar to the results previously reported in the literature. ${ }^{18}$ The molecular ion at $m / z=315$ was suitable for the identification of isorhamnetin (Table 1).

\section{Validation data}

All the references standards (vitexin, orientin, rutin, nicotiflorine, hyperoside, and isorhamnetin) showed linearity $\left(r^{2}=0.985\right.$, $0.999,0.951,0.9437,0.975$, and 1 , respectively) in a relatively different and wide concentration range. The RSD values of the inter-day precision experiments were $1,1.88,1.35,2.14,2.11$, and $2.32 \%$, respectively, and the intra-day variations were $2.04,1.01$, $1.10,2.67,1.89$, and $2.11 \%$, respectively. Their average regression equations were: $y=0.119+462785 x$ (vitexin); $y=0.369+$ $137922 x$ (orientin); $y=0.5612 x+0.0457$ (rutin); $y=0.3393 x-$ 0.6307 (nicotiflorine); $y=0.0851 x+0.1497$ (hyperoside); and $y=$ $1.7831 x-0.1302$ (isorhamnetin). The accuracy was determined by calculating the recovery values for each of the quantified flavonoids and was found to be $96.65 \pm 0.64 \%, 96.53 \pm 3.22 \%, 98.21 \pm 2.45 \%$, $99.34 \pm 2.23 \%, 96.89 \pm 3.43 \%$, and $94.45 \pm 1.34 \%$, respectively. The limits of detection were 7.14 (vitexin), 2.22 (orientin), 5.46 (rutin), 7.58 (hyperoside), 5.21 (nicotiflorine), and 5.83 (isorhamnetin). The identified compounds in the extracts of the floral parts of $M$. grandiflora L. were quantified by HPLC-PDA. The results (Table 2) show that the stamen extract contained the largest amounts of the identified flavonoids (ca. $140.6 \mathrm{mg} \mathrm{g}^{-1}$ ). Orientin was present in the highest concentration (ca. $66 \mathrm{mg} \mathrm{g}^{-1}$ ) and was mainly concentrated in the gynoecium. To the best of our knowledge, this study represents the first report of the presence of these flavonoids in the stamen, gynoecium, and petal of the M. grandiflora $\mathrm{L}$.

In the case of quantitative determination of total polyphenols, the linear regression analysis was $y=0.0925 x-0.0244$ (gallic acid), $r^{2}=$ 0.992 . The polyphenols content of the stamen was the richest $(0.7359$ $\pm 0.010 \mathrm{mg} \mathrm{g}^{-1}$ dry weight $)$, followed by that of the petal $(0.429 \pm$ $0.012 \mathrm{mg} \mathrm{g}^{-1}$ dry weight $)$ and gynoecium $\left(0.2890 \pm 0.022 \mathrm{mg} \mathrm{g}^{-1}\right.$ dry weight). The reported results are the means of three determinations.

Toxicological studies revealed that the ethanolic extracts of the organs under investigation were safe in a dose up to $5.7 \mathrm{~g} \mathrm{~kg}^{-1}$ body weight and can therefore be considered safe. ${ }^{19}$

\section{In vitro antioxidant effect}

Results of the in vitro antioxidant activity (Table 3 ) based on DPPH scavenging radical activity showed significant results for all the plant samples compared with that of the silymarin reference; EES was the most significant, with $67.26 \%$ potency, followed by EEG (52.71\%) and EEP (47.14\%). 
Table 1. MW, $\mathrm{R}_{t}, \mathrm{UV}$, and $\mathrm{MS}_{2}$ in the negative mode of the flavonoids in the ethyl acetate extract of M. grandiflora

\begin{tabular}{ccccccc}
\hline No & Compound & MW & $\begin{array}{c}{[\mathrm{M}-\mathrm{H}]^{-}} \\
\mathrm{m} / z\end{array}$ & $\begin{array}{c}\mathrm{R}_{\mathrm{t}} \\
\mathrm{min} .\end{array}$ & UV max & Precursor ions [m/z], MS $_{2}$ \\
\hline 1 & Apigenin 8- $C$-glucoside & 432 & 431 & 3.60 & 268,335 & $311(100 \%), 341(30 \%)$ \\
2 & Luteolin 8- $C$-glucoside & 448 & 447 & 4.96 & $256,269,350$ & $\begin{array}{c}327(100 \%), 357(70), 428(\mathrm{M}-18), \\
343(60 \%), 373(45 \%)\end{array}$ \\
& & & & & & $301(100 \%)$ \\
3 & Quercetin-3- $O$-rutinoside & 610 & 609 & 5.61 & $257,267 \mathrm{sh}, 359$ & $447(100 \%), 301(30 \%)$ \\
4 & Quercetin 3- $O$-galactoside & 464 & 463 & 6.97 & $253,260 \mathrm{sh}, 350$ & $301(30 \%)$ \\
5 & Quercetin 3- $O$-glucoside & 464 & 463 & 8.94 & $253,260 \mathrm{sh}, 350$ & 315 \\
6 & Kaempferol 3- $O$-rutinoside & 594 & 593 & 9.38 & 264,346 & 477,315 \\
7 & Isorhamnetin 3- $O$-glucoside & 478 & 477 & 9.74 & $258,305,361$ & 315,300 \\
8 & Isorhamnetin & 316 & 315 & 13.96 & $253,267 \mathrm{sh}, 306 \mathrm{sh}, 372$ & \\
\hline
\end{tabular}

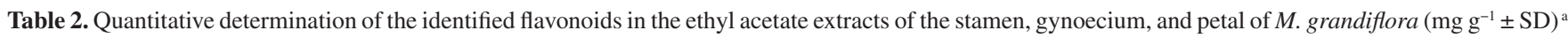

\begin{tabular}{ccccc}
\hline No & Compound & Stamen & Gynoecium & Petal \\
\hline 1 & Apigenin 8- $C$-glucoside & $\mathrm{nq}$ & $0.128 \pm 0.002$ & $2.89 \pm 0.005$ \\
2 & Luteolin 8- $C$-glucoside & $18.65 \pm 0.021$ & $66.89 \pm 0.035$ & $17.56 \pm 0.021$ \\
3 & Quercetin 3- $O$-rutinoside & $23.24 \pm 0.030$ & $0.086 \pm 0.024$ & $5.16 \pm 0.052$ \\
4 & Quercetin 3-O-galactoside & $11.21 \pm 0.002$ & $0.156 \pm 0.041$ & $3.12 \pm 0.002$ \\
5 & Quercetin 3- $O$-glucoside & $31.41 \pm 0.042$ & $38.25 \pm 0.003$ & $0.42 \pm 0.004$ \\
6 & Kaempferol 3- $O$-rutinoside & $16.55 \pm 0.003$ & $0.034 \pm 0.011$ & $3.41 \pm 0.004$ \\
7 & Isorhamnetin 3- $O$-glucoside & $1.00 \pm 0.005$ & $\mathrm{nq}$ & $\mathrm{nq}$ \\
8 & Isorhamnetin & $38.54 \pm 0.033$ & $\mathrm{nq}$ & $\mathrm{nq}$ \\
\hline & Sum of the determined compounds & 140.6 & & 32.56 \\
\hline
\end{tabular}

nq: not quantified. ${ }^{a}$ Each result is the mean of three determinations \pm standard deviation.

Table 3. In vitro antioxidant effect of EES, EEG, and EEP of M. grandiflora compared with that of silymarin

\begin{tabular}{cc}
\hline Treatment & Change from control (\%) \\
\hline Control & 56.1 \\
Slymarin & 89.8 \\
EES & 67.26 \\
EEG & 52.71 \\
EEP & 47.14 \\
\hline
\end{tabular}

EES, ethanolic extracts of stamen; EEG, ethanolic extracts of gynoecium; EEP, ethanolic extracts of petal.

\section{Hepatoprotection effect}

The results in Table 4 reveal that the administration of $\mathrm{CCl}_{4}$ induced acute liver toxicity, as evident from the increased levels of AST, ALT, and ALP. However, administration of doses of the test extracts under investigation or silymarin significantly preserved biochemical changes during $\mathrm{CCl}_{4}$ intoxification and confirmed their potential hepatoprotection activity to regenerate liver parenchyma cells. The results showed that EES was the most potent; it induced percentage decreases in the AST, ALT, and ALP levels of 56.3, 43.2,

Table 4. Effect of EES, EEG, and EEP of M. grandiflora compared with that of silymarin on serum enzymes level in liver-damaged rats $(\mathrm{n}=6)$

\begin{tabular}{|c|c|c|c|c|c|c|}
\hline & Liver enzymes & Zero & $15 \mathrm{~d}$ & $72 \mathrm{~h}$ & $15 \mathrm{~d}$ & $\%$ change \\
\hline \multirow{3}{*}{ Control } & AST(u/L) & $29.4 \pm 0.9$ & $28.6 \pm 0.4$ & $78.4 \pm 5.1$ & $84.4 \pm 5.9^{\mathrm{ab}}$ & - \\
\hline & $\operatorname{ALT}(\mathrm{u} / \mathrm{L})$ & $31.6 \pm 1.1$ & $30.9 \pm 0.7$ & $88.9 \pm 6.1$ & $91.4 \pm 5.7^{\mathrm{b}}$ & - \\
\hline & ALP (KAU) & $6.8 \pm 0.1$ & $7.1 \pm 0.1$ & $28.9 \pm 1.8$ & $33.4 \pm 2.3^{\mathrm{b}}$ & - \\
\hline \multirow{3}{*}{ EES } & $\mathrm{AST}(\mathrm{u} / \mathrm{L})$ & $25.7 \pm 1.1$ & $28.1 \pm 0.6$ & $63.9 \pm 2.4$ & $38.9 \pm 1.3^{\mathrm{ab}}$ & 35.5 \\
\hline & $\operatorname{ALT}(\mathrm{u} / \mathrm{L})$ & $27.6 \pm 0.4$ & $27.1 \pm 0.3$ & $68.9 \pm 2.6$ & $30.1 \pm 1.7^{\mathrm{ab}}$ & 56.3 \\
\hline & ALP (KAU) & $7.1 \pm 0.1$ & $8.8 \pm 0.1$ & $19.2 \pm 0.8$ & $10.8 \pm 0.6^{\mathrm{b}}$ & 43.2 \\
\hline \multirow{3}{*}{ EEP } & AST(u/L) & $38.9 \pm 0.8$ & $29.8 \pm 0.7$ & $67.3 \pm 2.5$ & $39.1 \pm 1.7^{\mathrm{ab}}$ & 35.2 \\
\hline & $\operatorname{ALT}(\mathrm{u} / \mathrm{L})$ & $31.4 \pm 1.1$ & $30.7 \pm 0.9$ & $74.9 \pm 3.2$ & $48.2 \pm 2.1^{\mathrm{ab}}$ & 35.6 \\
\hline & ALP (KAU) & $7.4 \pm 0.1$ & $8.2 \pm 0.1$ & $20.7 \pm 0.6$ & $14.9 \pm 0.7^{\mathrm{ab}}$ & 28 \\
\hline \multirow{3}{*}{ EEG } & $\mathrm{AST}(\mathrm{u} / \mathrm{L})$ & $27.6 \pm 1.2$ & $30.8 \pm 1.1$ & $82.4 \pm 2.7$ & $56.8 \pm 2.1^{\mathrm{ab}}$ & 31.1 \\
\hline & $\operatorname{ALT}(\mathrm{u} / \mathrm{L})$ & $33.2 \pm 1.2$ & $33.5 \pm 1.1$ & $91.6 \pm 3.8$ & $63.2 \pm 3.4$ ab & 21.4 \\
\hline & ALP (KAU) & $7.2 \pm 0.1$ & $7.4 \pm 0.1$ & $24.9 \pm 41$ & $22.9 \pm 0.9^{\mathrm{b}}$ & 8 \\
\hline \multirow{3}{*}{ Silym } & AST(u/L) & $28.3 \pm 1.1$ & $30.6 \pm 0.9$ & $55.3 \pm 1.3$ & $32.9 \pm 0.6^{\mathrm{a}}$ & 40.5 \\
\hline & $\operatorname{ALT}(\mathrm{u} / \mathrm{L})$ & $27.8 \pm 0.5$ & $26.8 \pm 0.4$ & $56.2 \pm 1.8$ & $29.2 \pm 0.8^{a}$ & 48 \\
\hline & ALP (KAU) & $7.3 \pm 0.1$ & $6.9 \pm 0.1$ & $18.3 \pm 0.6$ & $6.9 \pm 0.1^{\mathrm{a}}$ & 62.3 \\
\hline
\end{tabular}

a Statistically significant from $72 \mathrm{~h}$ time at $\mathrm{p}<0.01$; ${ }^{\mathrm{b}}$ Statistically significant from zero time at $\mathrm{p}<0.01$; silym, silymarin; EES, ethanolic extracts of stamen; EEG, ethanolic extracts of gynoecium; EEP, ethanolic extracts of petal. 
and $35.2 \%$, respectively.

The results of this investigation reveal that the stamen extract exhibited a protective effect against oxidative liver damage because of its high flavonoid content, which is not affected by the oxidative enzymes that induce a certain change in the color of other floral parts during drying or grinding ${ }^{1}$ and, consequently, may affect their constituents.

\section{CONCLUSION}

An investigation of the stamens, gynoecia, and petals of the flower of M. grandiflora was conducted for the first time and led to the identification of six flavonols in addition to vitexin and orientin. The ethanolic extracts of the organs under investigation exhibited bioactivities when examined for their antioxidant and hepatoprotection effects. The stamen extract exhibited a higher potency because of its total flavonoid content; these flavonoids exhibit hydrogen-donating-antioxidant activity and the ability to bind divalent metal cations.

\section{REFERENCES}

1. Sato, M.; Hiraoka, M.; Watanabe, A.; Phytochemistry 1992, 31, 3413.

2. Del Valle, M. L.; Tenorio, L. F. A.; Torres, N. J. C.; Zarco, O. G.; Pastelín, H. G.; Phytother. Res. 2009, 23, 666.

3. Guerra, B. L.; Alvarez, R. R.; Salazar, A. R.; Torres, C. A.; Rivas, G. V. M.; Waksman, T. N.; González, G. G. M.; Pérez, L. L. A.; Nat. Prod. Commun. 2013, 8, 135.
4. Báez, D.; Pino, J. A.; Morales, D.; Nat. Prod. Commun. 2012, 7, 237.

5. Farag, M. A.; Al-Mahdy, D. A.; Nat. Prod. Res. 2013, 27, 1091.

6. Jimenez, M. D.; Cordero, G. A.; Rojas, L. B.; Rodriguez, A. M.; Abstract Book of XXI Italo Latin American Congress of Ethnomedicine; Revista de la Facultad de Farmacia 2007, 49, 1.

7. Mohamed, S. M.; Ibrahim, N. A.; Ali, M. A.; Faraid, M. A.; $54^{\text {th }}$ Annual Congress of Medicinal Plants, Book of Abstracts Plant. Med.; University of Helsinki, Finland 2006, 72, 113.

8. Garg, S.; Kumar, S.; J. Essent. Oil Res. 1999, 11, 633.

9. Leung, A. Y.; In Chinese Medicinals; Janick, J.; Simon, J. E., eds.; Advances in new crops. Timber Press: Portland, 1990, 499.

10. Mellado, V.; Chavez, S. M. A.; Lozoya, X.; Arch. Invest. Med. (Mex) 1980, 11,335

11. Huang, H. C.; Hsieh, W. Y.; Niu, Y. L.; Chang, T. M.; BMC Complementary Altern. Med. 2012, 12, 72.

12. Mruthunjaya, K.; Hukkeri, V. I.; Pharmacogn. Mag. 2008, 4, 42.

13. Lorke, D.; Arch. toxicol. 1983, 275.

14. Williams, W. B.; Cuvelier, M. E.; Berset, C.; Lebensm. Wiss. Technol. 1995, 28, 25.

15. Klassen, C. D., Plaa, G. L.; Biochem. Pharmacol. 1969, 18, 2019.

16. Cuyckens, F.; Claeys, M.; J. Mass Spectrom. 2004, 39, 1.

17. El-Hawary, S. A.; Sokkar, N. M.; Ali, Z. Y.; Yehia, M. M.; J. Food Sci. 2011, 76, 1195.

18. Schieber, A.; Keller, P.; Streker, P.; Klaiber, I.; Carle, R.; Phytochem. Anal. 2002, 13, 87.

19. Buck, W. B.; Osweiter, G. D.; Van Gelder, A. G.; Clinical and Diagnostic Veterinary Toxicology $2^{\text {nd }}$ ed., 52011, Kendall/Hund Publishing Company: Iowa, 1976. 